\title{
Chronic Knee Dislocation and Flexion Contracture Treated with Open Reduction and External Fixation-A Case Report
}

\author{
Max Lingamfelter BS ${ }^{3}$, Hugo B Sanchez ${ }^{2}$ and Russell A Wagner ${ }^{1 *}$ \\ ${ }^{1}$ Associate Professor, Department of Orthopaedic Surgery, University of North Texas Health Science Center, Texas, USA \\ ${ }^{2}$ Assistant Professor, Department of Orthopaedic Surgery, University of North Texas Health Science Center, Texas, USA \\ ${ }^{3}$ Medical Student, University of North Texas Health Science Center-Texas College of Osteopathic Medicine, Texas, USA
}

\begin{abstract}
A 24 year old man presented 18 months after posterior knee dislocation, complaining of pain and inability to extend the knee or walk. An open reduction with posterior hamstring, gastrocnemius, and capsular release was performed and an anterior external fixator was placed to maintain the reduction for 3 weeks. A hinged external fixator was then placed for 4 weeks to maintain the reduction. No ligamentous reconstruction was performed. At one year after the initial operation, the patient had no pain, 5-120 degrees of motion, no clinical instability, and was able to walk. This case is an uncommon clinical scenario, the treatment was relatively straightforward and we were very happy with the result.
\end{abstract}

Keywords: Knee; Orthopaedic injuries; Knee arthroplasty; Ankle foot orthosis

\section{Introduction}

Chronic knee dislocation is an exceedingly rare and complicated disorder. Due to the scarcity and diversity of reported cases, there is no consensus regarding the best method of treatment [1-5]. The two major goals are to return stability to the joint while also providing range of motion (ROM) [4-6]. The use of a hinged external fixation device has emerged recently as a means of addressing both of these concerns $[2,7-$ 9].

To date there have been three previously reported cases that were similar in regards to the injury and treatment, except the case reported here had a much greater interval between injury and treatment. Simonian reported two cases that were reduced and reconstructed 4 months and 8 months after low velocity injury, followed by the application of a hinged external fixator. Thiel presented a case that used a very similar treatment method 3 months following a serious motor vehicle accident. Open reduction, soft tissue release, ACL and PCL reconstruction, and external fixation were used [10].

We present the case of a patient who presented 18 months after a knee dislocation treated with open reduction, soft tissue releases, and static followed by hinged external fixator placement. The authors have obtained the patient's informed written consent for print and electronic publication of the case report.

\section{Case Report}

The patient is a 24 year old male who was involved in a serious motor vehicle accident and suffered many injuries including a right femur fracture, right distal humerus fracture, and a severe closed head injury. Furthermore, his right knee was grossly deformed and posteriorly dislocated on radiographs. His femur was nailed but his other orthopaedic injuries were treated nonoperatively because of his serious medical condition and questionable survivability. However, gradually he recovered and was transferred to a nursing home. 18 months after his injury, he presented to our clinic because he wanted to be able to walk, his knee would not extend, and he had moderate knee pain. His right elbow had "auto-fused" at $90^{\circ}$ and his hand was contracted. His speech was limited and he was accompanied by his mother, who was determined to see him improve.

On exam, the right knee range of motion was $90^{\circ}-120^{\circ}$ and painful, with apparent posterior dislocation of the tibia. (Figure 1a,1b) $\mathrm{He}$ had a foot drop with a $10^{\circ}$ plantar flexion contracture. Quadriceps, hamstring, and ankle plantar flexion function were intact. Long-term skin breakdown with granulation tissue was present on his abdomen, from a previous surgery, and on his right ischial tuberosity; and he had a suprapubic catheter. He weighed 260 pounds.

An open reduction was performed 18 months after the initial injury. A medial parapatellar approach was used, leaving the medial meniscus intact. The articular cartilage on the femur, tibia and patella were in fairly good condition and the ACL and PCL was missing. All scar tissue was released in the anterior knee. With the knee in flexion, the tibia could be forcibly reduced by placing a Hohmann retractor, padded with a laparotomy sponge, against the distal femur with the tip of the retractor behind the proximal tibia in the intercondylar notch. However, the knee could then be extended to only a $60^{\circ}$ contracture. A posteromedial incision was made, the medial hamstrings were released, and the medial and lateral gastrocnemius and posterior capsule were elevated off the femur. With some force, the knee could be fully extended. A uniplanar external fixator was then applied to maintain reduction and hold the knee in extension. Figure $2 \mathrm{a}, 2 \mathrm{~b}$ Percutaneous achilles lengthening was also performed with continuance of the ankle foot orthosis. He began physical therapy at the nursing home, weightbearing as tolerated.

Three weeks following the reduction, the uniplanar external fixator was replaced with a "Compass" (Smith \& Nephew) hinged external

${ }^{*}$ Corresponding author: Dr. Russel Wagner, Vice Chairman Department of Orthopaedic Surgery, Program Director Orthopaedic Residency, John Peter Smith Hospital, Fort Worth, Texas, USA; E-mail: RWagner@jpshealth.org

Received February 17, 2014; Accepted June 06, 2014; Published June 16, 2014

Citation: Max Lingamfelter BS, Sanchez HB, Wagner RA (2014) Chronic Knee Dislocation and Flexion Contracture Treated with Open Reduction and External Fixation-A Case Report. J Arthritis 3: 133. doi:10.4172/2167-7921.1000133

Copyright: ( 2014 Max Lingamfelter BS, et al. This is an open-access article distributed under the terms of the Creative Commons Attribution License, which permits unrestricted use, distribution, and reproduction in any medium, provided the original author and source are credited. 


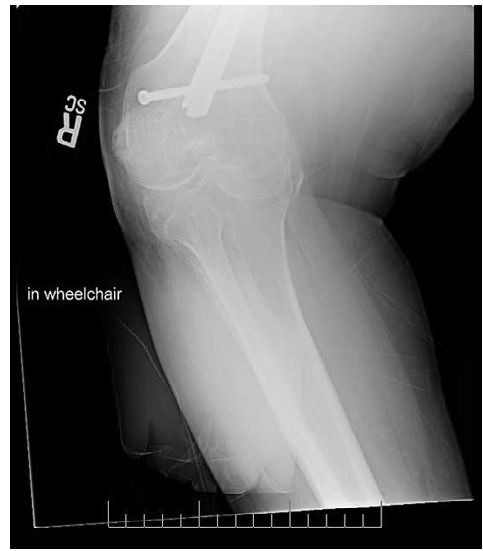

Figure 1a: Anteroposterior (AP) radiograph of the knee showing posterior dislocation.

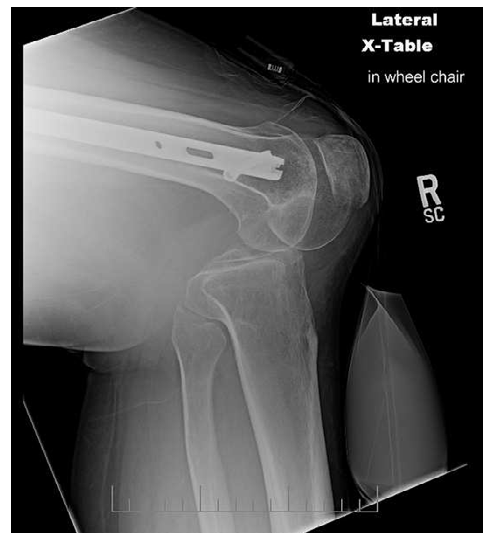

Figure 1b: Lateral radiograph of the knee showing posterior dislocation.

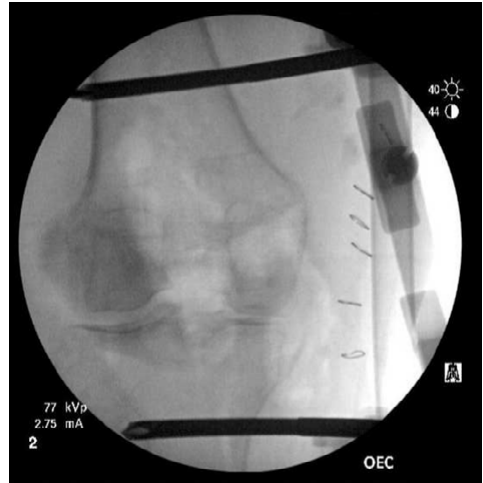

Figure 2a: AP radiograph of the knee showing reduction with the uniplanar fixator.

fixator to allow ROM and maintain reduction. Intraoperatively, a pin was placed in the rotational axis of the distal femur and the hinged external fixator was built around this pin. One half-ring was placed proximally and one half-ring distally with two pins in each. When it was verified that the knee would remain reduced in extension and flexion, the axis pin was removed. In the figure $3 a, 3 b$ posteromedial incision had an area of marginal wound necrosis that was debrided and negative pressure wound therapy was used.
The hinge broke and was removed a month following its placement; at this point the patient had achieved a ROM of $20^{\circ}-90^{\circ}$, the knee remained reduced and stable, and he rated his pain as 0 out of $10 . \mathrm{He}$ continued working with Physical Therapy at the nursing home. Five months following the open reduction, the patient was taken to the OR for repeat debridement and delayed wound closure of the posteromedial wound. Cultures showed Methicillin Sensitive Staphylococcus aureus, and following oral antibiotic treatment, the wound finally healed. His range of motion gradually improved so that at one year, he had near full extension and $120^{\circ}$ of flexion, and he could walk slowly with a platform walker. He did not complain of instability and his knee felt stable on exam. He was able to return home from the nursing home and he and his mother were very satisfied with the outcome.

\section{Discussion}

Chronic knee dislocation is an exceedingly rare disorder and therefore there is a lack of consensus regarding the preferred treatment method. The patient's goals were to extend his knee and regain mobility. To achieve this goal there were a few options. Knee motion helps maintain healthy articular cartilage, so there was concern that the cartilage would be damaged. In that case, possibly an arthrodesis or arthroplasty would be the best option. Arthrodesis was considered but probably would have resulted in less patient satisfaction, lower back pain, and permanent disability [11]. Total knee arthroplasty was used recently to address chronic knee dislocation in a 52-year-old woman who presented 27 months after being struck by a motor vehicle [11] That case represents the only case of chronic posterior knee dislocation with a longer delay between injury and treatment to our knowledge.

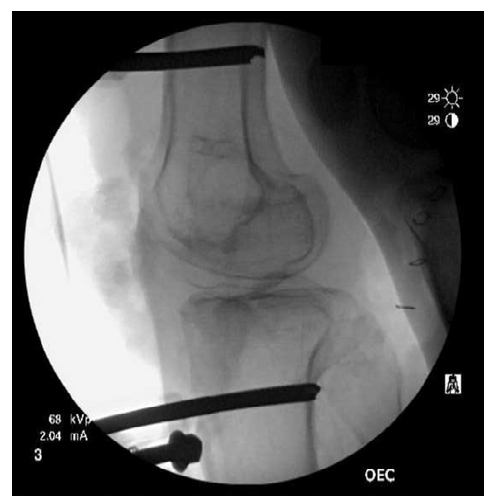

Figure $\mathbf{2 b}$ : Lateral radiograph of the knee showing reduction with the uniplanar fixator.

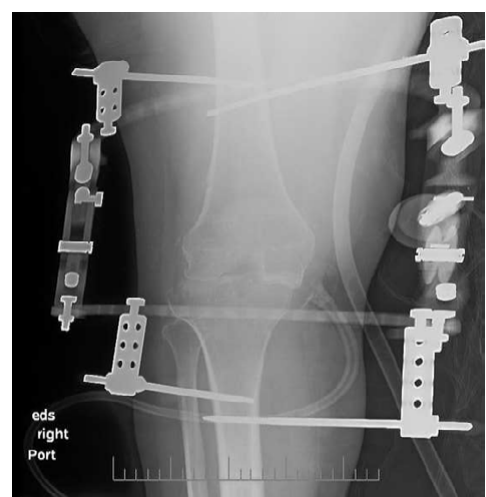

Figure 3a: AP radiograph of the knee showing the hinged external fixator 


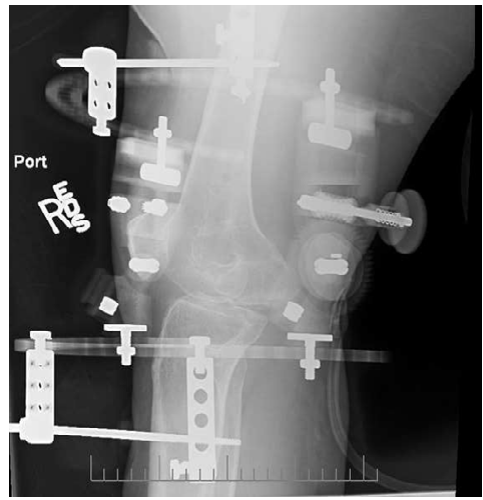

Figure 3b: Lateral radiograph of the knee showing the hinged external fixator.

At a two year follow up, the patient had achieved a range of motion of $0^{\circ}-85^{\circ}$ without pain or instability. In our patient, total knee arthroplasty may not have been a good choice because of the areas of chronic skin breakdown, his suprapubic catheter, his obesity, and his young age. At the time of the open reduction, it could be seen that his articular cartilage was not seriously degraded, although it is highly probable that he will develop arthritis in the future.

Using an external fixator has become a common method of addressing instability of the knee following knee dislocation $[2,7,8,12]$. It addresses the goals of providing stability and mobility; and a hinged external fixator will allow early ROM. Also, a hinged external fixator may reduce the risk of failure following reconstruction. Another potential option in this case would have been to use a cast such as reported by Henshaw [13]. A cast would have presented a reduced risk of pin tract infection and lower cost; however, our patient had a history of heel breakdown and this likely would have recurred. Also, the static external fixator provided greater force to maintain the extension gained in the operating room.

Three cases of chronic knee dislocation treated with a hinged external fixator have previously been reported. Two cases were reported by Simonian [7]. The first case presented three months following a low velocity injury. Following extensive releases, ACL and PCL reconstruction, and hinged external fixator application for 6 weeks, a final range of motion of $-5^{\circ}$ to $105^{\circ}$ was achieved at fifteen weeks. The second case was also following a low velocity injury that presented 8 months following injury and received similar treatment. Six months following treatment, her ROM was $0^{\circ}$ to $120^{\circ}$. Thiel reported a case that presented 3 months after a motor vehicle accident. 10 Significant releases were required, the ACL and PCL were reconstructed, and a long leg splint was used for ten days prior to the external fixator placement. At the 1.5 year followup, a final ROM of 0 to 90 degrees was achieved.

Our case differs from these due to the greatly extended time between injury and final treatment. There is consensus that extended periods of time between injury and treatment are correlated with a worse outcome, having a lower score on the Daily Living Scale and Sports activity Scale $[14,15]$. Longer periods of dislocation allow for considerable scar tissue and contracture to develop in soft tissues [1]. Additionally, in the posteriorly dislocated and flexed position, the posterior capsule and collateral ligaments will contract. Once the knee is reduced these structures can prevent full extension [3]. It has been suggested that use of a CPM machine, over a period of 2 to 3 weeks pre-operatively, could address these concerns of arthrofibrosis [16]. Additionally, high-energy injuries have a significantly less favorable outcome when compared with low-energy injuries $[17,18]$. Patients with multiple traumatic injuries have also been noted to have a less favorable outcome due to limited or absent early rehabilitation. $[8,19,20]$. Overall, chronic high energy knee dislocations are a very small subset of an already rare orthopedic occurrence [17]. For this reason, a results-based analysis of treatment protocols is limited $[2,3,21,22]$.

\section{Conclusion}

Chronic Knee dislocation is a rare phenomenon that necessitates difficult decisions from the patient and physician. The final outcome will be worsened the longer the joint is dislocated; therefore, it is important to diagnose and reduce knee dislocations as soon as possible if the patient's status permits. Delay in reduction presents a difficult situation for the physician further down the road as arthrofibrosis and contracture commonly complicate the picture. Treatment options vary from nonoperative to reduction with possible reconstruction to total knee arthroplasty. The lack of consensus in this area makes the treatment plan very individualized and relies heavily on the judgment of the physician. In this patient, even 18 months after the injury, the cartilage was viable and the knee could be reduced and function improved.

\section{References}

1. Rihn JA, Groff YJ, Harner CD, Cha PS (2004) The acutely dislocated knee: evaluation and management. J Am Acad Orthop Surg 12: 334-346.

2. Marcacci M, Zaffagnini S, Bonanzinga T, Pizzoli A, Manca M, et al. (2012) Surgical technique: Articulated external fixator for treatment of complex knee dislocation. Clin Orthop Relat Res 470: 869-876.

3. Petrie RS, Trousdale RT, Cabanela ME (2000) Total knee arthroplasty for chronic posterior knee dislocation: Report of 2 cases with technical considerations. J Arthroplasty 15: 380-386.

4. Ríos A, Villa A, Fahandezh H, de José C, Vaquero J (2003) Results after treatment of traumatic knee dislocations: a report of 26 cases. J Trauma 55: 489-494.

5. Harner CD, Waltrip RL, Bennett CH, Francis KA, Cole B, et al. (2004) Surgica management of knee dislocations. J Bone Joint Surg Am 86-86A: 262-73.

6. Howells NR, Brunton LR, Robinson J, Porteus AJ, Eldridge JD, et al. (2011) Acute knee dislocation: an evidence based approach to the management of the multiligament injured knee. Injury 42: 1198-1204.

7. Simonian PT, Wickiewicz TL, Hotchkiss RN, Warren RF (1998) Chronic knee dislocation: reduction, reconstruction, and application of a skeletally fixed knee hinge. A report of two cases. Am J Sports Med 26: 591-596.

8. Stannard JP, Sheils TM, McGwin G, Volgas DA, Alonso JE (2003) Use of hinged external knee fixator after surgery for knee dislocation. Arthroscopy 19: 626-631.

9. Eastwood B, Albers HW, Albert M (2008) Knee dislocation in a 9-year-old boy. Am J Orthop (Belle Mead NJ) 37: E110-112.

10. Van Thiel GS, Baker CL 3rd, Bush-Joseph C (2009) A chronic posterolatera knee and patella dislocation: case report. J Orthop Trauma 23: 541-545.

11. Liporace FA, Hommen JP, Su ET, Jeong GK, Dayan AJ (2006) Semiconstrained knee arthroplasty in the setting of a chronic knee dislocation: a case report. J Orthop Trauma 20: 286-288.

12. Sommers MB, Fitzpatrick DC, Kahn KM, Marsh JL, Bottlang M (2004) Hinged external fixation of the knee: intrinsic factors influencing passive joint motion. $J$ Orthop Trauma 18: 163-169.

13. Henshaw RM, Shapiro MS, Oppenheim WL (1996) Delayed reduction of traumatic knee dislocation. A case report and literature review. Clin Orthop Relat Res: 152-156.

14. Levy BA, Dajani KA, Whelan DB, Stannard JP, Fanelli GC, et al. (2009) Decision making in the multiligament-injured knee: an evidence-based systematic review. Arthroscopy 25: 430-438. 
Citation: Max Lingamfelter BS, Sanchez HB, Wagner RA (2014) Chronic Knee Dislocation and Flexion Contracture Treated with Open Reduction and External Fixation-A Case Report. J Arthritis 3: 133. doi:10.4172/2167-7921.1000133

15. Harner CD, Waltrip RL, Bennett CH, Francis KA, Cole B et al. (2004) Surgical management of knee dislocations. J Bone Joint Surg Am 86: 262-263.

16. Ibrahim SA, Ahmad FH, Salah M, Al Misfer AR, Ghaffer SA, et al. (2008) Surgical management of traumatic knee dislocation. Arthroscopy 24: 178-187.

17. Engebretsen L, Risberg MA, Robertson B, Ludvigsen TC, Johansen S (2009) Outcome after knee dislocations: a 2-9 years follow-up of 85 consecutive patients. Knee Surg Sports Traumatol Arthrosc 17: 1013-1026.

18. Shelbourne KD, Porter DA, Clingman JA, McCarroll JR, Rettig AC (1991) Lowvelocity knee dislocation. Orthop Rev 20: 995-1004.

19. Shapiro MS, Freedman EL (1995) Allograft reconstruction of the anterior and posterior cruciate ligaments after traumatic knee dislocation. Am J Sports Med 23: $580-587$.

20. Wascher DC, Becker JR, Dexter JG, Blevins FT (1999) Reconstruction of the anterior and posterior cruciate ligaments after knee dislocation. Results using fresh-frozen nonirradiated allografts. Am J Sports Med 27: 189-196.

21. Levy BA, Fanelli GC, Whelan DB, Stannard JP, MacDonald PA, et al. (2009) Controversies in the treatment of knee dislocations and multiligament reconstruction. J Am Acad Orthop Surg 17: 197-206.

22. Robertson A, Nutton RW, Keating JF (2006) Dislocation of the knee. J Bone Joint Surg Br 88: 706-711. 